LAURIE M. BROWN

della Northwestern Oniversity

\title{
PERTURBATION THEORY AT HIGH ENERGY
}

(Conferenza tenuta il 13 maggio 1967)*

\section{SUMMARY ( $\left.{ }^{(}\right)$}

Methods are presented for simplifying the algebraic structure of quantum electrodynamics perturbation amplitudes in the framework of the two-component fermion theory, especially useful in the high energy limit. The simplification is accomplished by the use of two rules:

Rule $I$ is proved in general and gives a prescription for the elimination of all «double corner graphs, which otherwise appear in the two-component formalism in the same form as they appear in the Klein-Gordon or Pauli-Weisskopf theories. The rule states that "double corner 》 terms can be omitted providing that quantities $p_{i}{ }^{2}$, where $p_{i}$ is an internal four momentum, appearing in the numerators of Feynman graph expressions are replaced by $m_{i}{ }^{2}$, where $m_{i}$ is the mass of the internal fermion line.

Rule II, which is valid only in the extreme relativistic limit, says that amplitude is dominant which conserves cohelicity (defined as helicity for particles and negative helicity for antifermions) along the fermion line. It further gives a prescription for writing a simplified cohelicity preserving amplitude.

In addition to stating and proving the two rules, methods are presented for the systematic evaluation of matrix elements, rather than their absolute squares summed over spins, by the use of trace techniques. These are generally simpler to apply than the usual techniques and yield substantially more physical information.

* Pervenuta in tipografia il 20 giugno 1967.

( ${ }^{1}$ Based on « Perturbation Theory at High Energy 》 by Laurie M. Brown and Ik-Ju Kang (to be published in Nuovo Cimento). 